JOANNA KOTOWSKA

Agata RęBKowsKa

Université de Wrocław

\title{
VERS UNE CARTOGRAPHIE DES RECHERCHES PHILOLOGIQUES : OBSERVATIONS À L'OCCASION DU 50 ĖME ANNIVERSAIRE DE LA REVUE ROMANICA WRATISLAVIENSIA
}

\section{INTRODUCTION}

Le cinquantième anniversaire de la revue Romanica Wratislaviensia est certainement une bonne occasion pour faire des bilans. En 50 ans d'existence, la revue a subi plusieurs transformations d'ordre formel et conceptuel. Sur les pages de Romanica s'inscrivent les chemins qu'ont suivis au cours des années les romanistes de Wrocław et d'autres centres de recherche. La revue témoigne non seulement des centres d'intérêt des chercheurs, mais permet aussi de retracer leurs parcours professionnels et parfois même, comme un journal intime, de révéler des histoires personnelles... Mais c'est surtout la variété des thématiques abordées — dont témoignent d'ailleurs de nombreuses publications ${ }^{1}$ — qui nous invite à esquisser le landscape ${ }^{2}$

${ }^{1}$ Comme, par ex., celles de E. Kulak (« Między komparatystyką i przekładoznawstwem : romanistyka wrocławska w latach powojennych », [dans :] Język - Stereotyp - Przekład, E. Skibińska et M. Cieński (dir.), Dolnośląskie Wydawnictwo Edukacyjne, Wrocław 2002, pp. 15-24) ou de J. Jakubowska-Cichoń et N. Paprocka (« Sous le signe de l'interdisciplinarité : recherches en littérature et linguistique françaises à l'Université de Wrocław au début du XXI siècle », [dans :] Les études françaises dans les pays de Visegrád : histoire, présent et avenir, T. Jaroszewska (dir.), Oficyna Wydawnicza LEKSEM, Łask 2013, pp. 49-58), dont nous reparlerons plus tard.

${ }^{2}$ La métaphore de landscape utilisée pour parler d'une discipline ou aire de recherche est empruntée à Federico Zanettin, Gabriela Saldanha \& Sue-Ann Harding, « Sketching landscapes in translation studies: A bibliographic study », Perspectives, vol. 23:2, 2015, pp. 161-182. 
des recherches en philologie romane qui se dégage des contributions publiées dans la revue, depuis sa création en 1968, jusqu'au dernier numéro paru en 2016 (nous rédigeons cet article en juin 2017). Pour ce faire, nous allons observer ce réservoir d'idées qu'est Romanica afin de documenter les activités des contributeurs et de dresser une cartographie du paysage des recherches philologiques inscrit dans la revue ${ }^{3}$.

\section{OBJET DES RECHERCHES PHILOLOGIQUES}

Avant d'examiner la revue, regardons de plus près la définition de philologue telle qu'on la retrouve dans les dictionnaires. L'étiquette - quoique centrée sur l'humain - fournit également des informations sur l'objet de la recherche philologique.

Étymologiquement, le terme philologue renvoie à « celui qui aime les belles lettres ", à un " savant en matière de langue et de littérature » (TLFi). Il n'est pas sans importance que le sens accordé à la philologie en tant que discipline scientifique diffère selon l'époque et la zone géographique. Hans Ulrich Gumbrecht, auteur de The Powers of Philology: Dynamics of Textual Scholarship, adopte une définition classique du terme, proche des conceptions allemandes du XIX ${ }^{\mathrm{e}}$ siècle : pour lui, la philologie désigne l'ensemble des compétences académiques requises pour l'édition critique des textes historiques ${ }^{4}$. Une telle définition implique que l'intérêt de la philologie soit porté aux grands textes du passé, considérés comme plus importants que ceux de la culture contemporaine. La tâche du philologue consisterait donc à identifier et à restaurer des documents historiques, souvent fragmentaires et incomplets, à présenter la pluralité de leurs versions, ainsi qu'à en fournir un commentaire. La méthode du philologue, quant à elle, s'opposerait à l'herméneutique des textes, sensible à l'influence des intuitions du moment. Perçue dans un esprit cartésien, elle devrait se caractériser par l'objectivité, le rationalisme et la modération du chercheur ${ }^{5}$.

Comme l'observe Claire Rétat-Frydryszak, avec les influences des romantiques et du nationalisme dans l'Allemagne du XIX ${ }^{\mathrm{e}}$ siècle, l'étude des textes s'est étendue ensuite aux « textes post-antiques [...], puis aux textes en langue moderne,

${ }^{3}$ Pour réaliser ce travail, nous nous sommes inspirées de l'article de Gideon Toury qui esquisse une cartographie des recherches traductologiques sur l'exemple de la revue Target. Voir G. Toury, " Incubation, birth and growth. Observations on the first 20 years of Target », Target, vol. 21:2, 2009, pp. 189-207. Voir aussi E. Skibińska, « La gratitude des romanistes. Sur l'exemple de Romanica Wratislaviensia », [dans :] Être philologue. Mélanges offerts à Teresa Giermak-Zielińska, W. Fijałkowska, M. Izert, A. Kiliszczyk et al. (dir.), Wydawnictwa Uniwersytetu Warszawskiego, Warszawa 2015, pp. 211-223.

4 "Configuration of scholarly skills that are geared toward historical text curatorship » (H.U. Gumbrecht, The Powers of Philology. Dynamics of Textual Scholarship, University of Illinois Press, Champaign 2003, p. 4).

5 Ibidem, pp. 3-5. 
donnant naissance à la néophilologie ou philologie moderne $»^{6}$. Aujourd'hui, elle renvoie, du moins en Europe Centrale, à l'ensemble des méthodes appliquées à l'analyse des textes pour en établir le sens ou bien pour accéder à un savoir extralinguistique à travers les textes ${ }^{7}$. Cette définition pluridimensionnelle, qui prend en compte aussi bien l'analyse des faits de langage que des phénomènes littéraires, se trouve confirmée dans des dictionnaires allemands ${ }^{8}$ ou polonais ${ }^{9}$, qui semblent refléter le sens contemporain du terme en Europe Centrale.

La question qui se pose est donc de savoir dans quelle image du philologue s'inscrivent les recherches philologiques publiées dans Romanica Wratislaviensia: correspondent-elles à l'objet d'études du philologue au sens classique du terme, ou bien se rapprochent-elles des activités du philologue contemporain? Afin de trouver une réponse, dressons un répertoire des éléments qui contribuent à l'image des recherches philologiques inscrite dans Romanica. Les langues de travail, les types de contributions ainsi que les thématiques abordées devraient être révélateurs.

\section{LES AUTEURS DE ROMANICA WRATISLAVIENSIA ET LEURS RECHERCHES}

\subsection{LES PHILOLOGUES EN CHIFFRES}

L'observation des données quantitatives permet déjà de formuler de premières remarques sur l'identité du romaniste. Rappelons donc seulement que la revue a été créée en 1968 (tout d'abord dans le cadre de la série Acta Universitatis Wratislaviensis), qu'elle a été l'une des premières revues des romanistes polonais ${ }^{10}$, et qu'elle est depuis toujours publiée aux presses de l'Université de Wrocław.

${ }^{6}$ C. Rétat-Frydryszak, « La philologie romane en Europe Centrale et en France au XIX ${ }^{\mathrm{e}}$ siècle », Romanica Wratislaviensia LI, Wydawnictwo Uniwersytetu Wrocławskiego, Wrocław 2004, p. 76.

7 Ibidem, pp. $75-76$.

8 «Wissenschaft, die sich mit der Erforschung von Texten in einer bestimmten Sprache beschäftigt; Sprach- und Literaturwissenschaft » [Science qui s'occupe de l'étude des textes dans une langue particulière ; science de la langue et de la littérature], Duden [en ligne]: <http://www. duden.de/rechtschreibung/Philologie> [consulté le 2.08.2017]. Nous traduisons.

${ }^{9}$ URL : <https://sjp.pwn.pl/szukaj/filologia.html> [consulté le 2.08.2017].

${ }^{10}$ La même année paraît Acta Philologica, revue publiée par la Faculté de Néophilologie de l'Université de Varsovie. Cependant, son profil diffère considérablement de celui de Romanica, la revue publiant des travaux qui s'inscrivent dans le cadre de plusieurs philologies, pas seulement romane. Une autre revue publiée à partir de 1968 est Acta Universitatis Lodziensis. Folia Litteraria, celle-ci étant à l'origine restreinte aux contributions en littérature. Studia Romanica Posnaniensia, dont l'histoire commence en 1971, est une revue de statut semblable à celui de Romanica, c'est-àdire qui rassemble les sujets linguistiques et littéraires. Parmi les titres plus récents (apparus dans les années 2000), on peut citer par ex. Romanica Cracoviensia (2000) ou Romanica Silesiana (2006). 


\subsubsection{CONTRIBUTEURS}

En près d'un demi-siècle d'existence, Romanica ont publié les travaux de 449 chercheurs. La plupart d'entre eux (263 personnes) n'ont soumis à la revue qu'un seul article, ce qui se laisse interpréter comme un signe d'hétérogénéité et de regard bienveillant des rédacteurs, qui semblent prêts à accueillir facilement de nouvelles voix. Mais il y a aussi des noms qui reviennent systématiquement : 163 auteurs ont publié 2 à 3 articles, 31, 4 à 9 , six personnes, 10 à 15 , et trois " champions » en ont publié 16 à 20. Sans surprise, ce sont surtout des chercheurs wratislaviens : sur les 727 affiliations qui accompagnent les articles ${ }^{11}, 368$ correspondent aux centres de recherche de Wrocław. Il est à noter que $71 \%$ des contributeurs sont des représentants des universités polonaises. Le caractère de la revue n'est cependant pas strictement national; la (les) langue(s) de travail (voir 3.1.3.) et le nombre de chercheurs d'universités étrangères qui y ont participé en apportent la preuve. Ces derniers représentent $29 \%$ de l'ensemble. Pour ce qui est des affiliations étrangères, les centres de recherche français sont largement majoritaires, ce qui reflète bien le caractère fort des relations franco-polonaises. Les autres pays européens (et a fortiori non-européens) sont beaucoup moins représentés, comme le montre le graphique 1 :

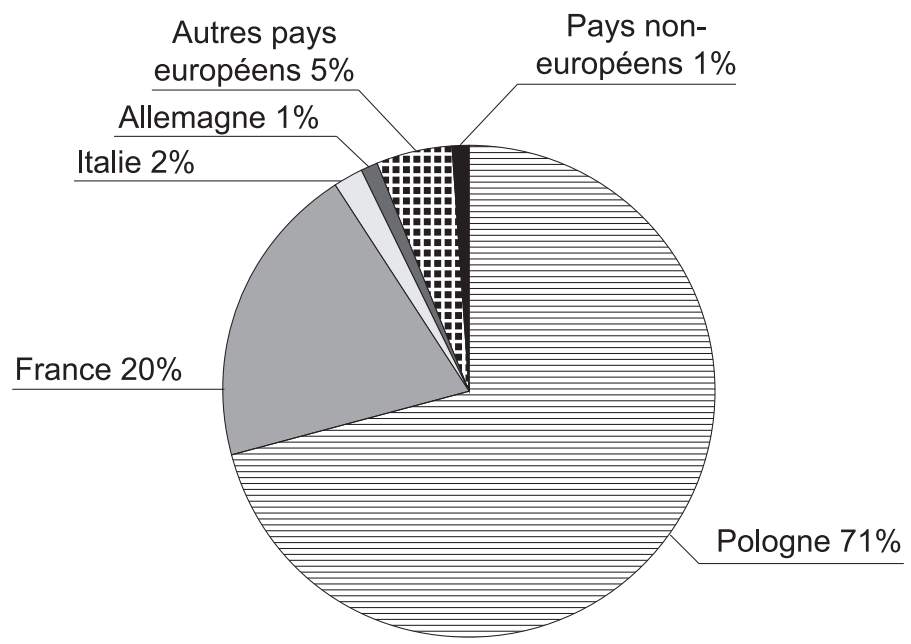

Graphique 1. Les contributeurs de Romanica Wratislaviensia par pays Source : Recherche personnelle des auteures.

L'observation des noms des contributeurs et de leurs affiliations permet de constater encore une chose : être romaniste ne veut pas dire nécessairement être

${ }^{11}$ Les affiliations ne commencent à être signalées de façon régulière qu'à partir du numéro XVII. Elles ne sont pas indiquées dans les numéros I à XI, et dans les numéros XII à XVI, elles n'accompagnent que certains articles. 
rattaché à un seul organisme de recherche. En effet, certains noms reviennent systématiquement tout au long des années, ce qui semble refléter une certaine continuité de la revue. Mais il y en a aussi d'autres qui disparaissent à un moment donné ${ }^{12}$ pour réapparaître quelque temps plus tard avec une autre affiliation institutionnelle (il s'agit surtout des trois personnes qui ont quitté l'Université de Wrocław pour s'installer dans d'autres villes polonaises ou françaises). De l'autre côté, certaines personnes $(9$ cas) sont rattachées à plusieurs centres de recherche.

\subsubsection{CONTRIBUTIONS}

Au début de son existence, le statut particulier de la revue - publiée dans le cadre d'une série - facilitait la publication d'études à caractère très hétérogène. On pouvait y retrouver par exemple des actes de colloques, mais aussi des ouvrages monographiques. Au fil du temps, la superposition de différents facteurs socio-politiques - pour n'en citer que quelques-uns, le changement de régime ou la mise en place de nouvelles législations concernant les revues scientifiques - a abouti à restreindre le profil de Romanica Wratislaviensia. Aujourd'hui, elle a le statut de périodique scientifique.

$\mathrm{Au}$ total, la revue compte soixante-quatre numéros. On y retrouve des numéros thématiques (certains sont des fruits des colloques organisés par l'Université de Wrocław souvent en collaboration avec d'autres centres de recherche), mais aussi des ouvrages monographiques et des mélanges offerts aux chercheurs de mérite.

Ces données appellent néanmoins quelques précisions. La première est liée à l'âge et au parcours professionnel des contributeurs. Sans examiner les actes de naissance des auteurs, on observe que la revue ouvre son espace d'expression aussi bien aux jeunes chercheurs qu'à leurs collègues plus expérimentés. Ces derniers, tout d'abord auteurs de thèses de doctorat publiées sous forme d'ouvrages monographiques ${ }^{13}$, sont devenus au fil du temps des maîtres pour leurs futurs disciples. Ce rayonnement de la démarche des maitres contribue à la continuité de la revue, car il se retrouve dans les thématiques des recherches abordées par leurs élèves ${ }^{14}$.

${ }^{12}$ Les raisons varient, mais la plus évidente est la fin de carrière.

${ }^{13}$ Les ouvrages monographiques (y compris les thèses de doctorat) publiés dans la revue sont de Mieczysława Sekrecka, Anna Nikliborc, Józef Heistein (deux ouvrages), Aleksander Labuda, Krystyna Gabryjelska, Eugeniusz Ucherek, Elżbieta Skibińska et Edmund Sikora.

${ }^{14}$ Le « rayonnement » de la démarche est visible dans de nombreux articles publiés dans la revue. Citons à titre d'exemple ceux qui reprennent la méthode d'Eugeniusz Ucherek : Elżbieta Biardzka, «Les syntagmes français régis par les prépositions sans et avec dans la traduction polonaise » (Romanica Wratislaviensia XXXIX, 1992), «Les syntagmes français régis par hors (de) exprimant l'exception dans la traduction en polonais » (Romanica Wratislaviensia XLI, 1996); Witold Ucherek, «La traduction en polonais de quelques locutions prépositives françaises construites avec bout » (Romanica Wratislaviensia XLIII, 1997), « La construction $\grave{a}+$ adjectif + coups + de dans la traduction polonaise » (Romanica Wratislaviensia LI, 2004), ou dans la lignée de la littérature 
La deuxième remarque concerne les actes de colloque. On observe que la plupart des actes (18) ont été publiés dans la période comprise entre la seconde moitié des années 1970 et le début des années 1990. Onze de ces recueils sont le fruit de la collaboration de l'Université de Wrocław avec des centres de recherche étrangers (surtout dans le cadre de contrats bilatéraux avec La Sorbonne Nouvelle ou l'Université Charles-de-Gaulle de Lille 3, qui seront à l'origine de publications systématiques dans les années 1980). Cela montre que malgré les circonstances politiques défavorables de l'époque communiste, malgré le régime hostile à toute forme de contacts des citoyens polonais avec l'Occident, les romanistes polonais participaient activement à la vie intellectuelle de la communauté internationale, ce qui facilitait sans doute l'internationalisation de la recherche ${ }^{15}$. Actuellement, la revue s'ouvre toujours aux contributions présentées lors de colloques internationaux (comme ceux dédiés à la traduction comme moyen de communication interculturelle), mais depuis le début des années 2000, elle publie surtout des numéros thématiques consacrés à une problématique annoncée dans les appels à contributions.

\subsubsection{LANGUE(S) DE TRAVAIL}

L'identité du romaniste semble également déterminée par sa langue d'expression. Dans le cas de Romanica, sur 736 contributions au total (sans compter les comptes-rendus ou les résumés des communications présentées lors de colloques), 612 (soit plus de 83\%) ont été écrites en français. Ce résultat est d'autant plus révélateur que $71 \%$ des contributeurs sont des universitaires polonais ${ }^{16}$. Ce qui frappe, c'est la présence très faible de l'espagnol $(5 \%)$ et de l'italien $(4,8 \%)$ ainsi que l'absence des autres langues romanes (voir le graphique 2). Il est aussi à noter que seuls six auteurs ont publié dans Romanica en deux langues romanes (dont le français). Cela montre l'évolution du profil de recherche du philologue, qui devient spécialiste d'une seule langue, quel que soit l'objet de sa recherche. En outre, la présence prédominante du français s'explique par la création à l'Institut des Études Romanes de l'Université de Wrocław de deux autres revues destinées aux chercheurs s'exprimant en espagnol et en italien (Estudios Hispánicos et Italica Wratislaviensia), en 1988 et 2010.

\footnotetext{
comparée, courant initié à l'Institut d'Études Romanes de Wrocław par Józef Heistein : Małgorzata Tomicka, "La critique du Symbolisme français en Pologne avant la première guerre mondiale » (Romanica Wratislaviensia XVIII, 1986) ; Justyna Łukaszewicz, «Entre l'Amphitryon de Molière et l'Amfitrio de Zabłocki» (Romanica Wratislaviensia XLVIII, 2002), etc.

${ }^{15}$ Celle-ci étant l'une des conditions de l'intégration internationale de la discipline scientifique et de son ouverture au modèle universaliste des sciences humaines (voir W. Kalaga, « Inte/gracja a granice dyscypliny », Postscriptum Polonistyczne 9, 2012, pp. 25-26).

${ }^{16}$ Compte tenu de la remarque de la note 12 , ce résultat ne peut être qu'approximatif. Le score est obtenu en analysant les 727 affiliations.
} 


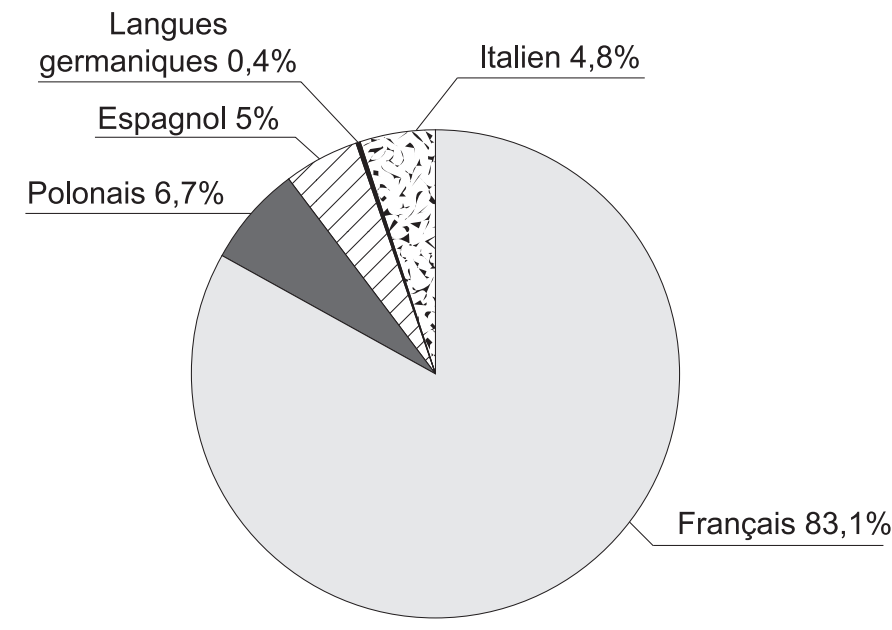

Graphique 2. Langues des contributions de Romanica Wratislaviensia Source : Recherche personnelle des auteures.

\subsubsection{THÉMATIQUES DE RECHERCHE}

Les thématiques de recherche abordées par les chercheurs de l'Institut d'Études Romanes de Wrocław ont été présentées dans les travaux d'Ewa Kulak ${ }^{17}$, Joanna Jakubowska-Cichoń et Natalia Paprocka ${ }^{18}$ ou Witold Ucherek $^{19}$. Les auteurs montrent les principales directions de recherche, telles que les études de littérature des $\mathrm{XVII}^{\mathrm{e}}, \mathrm{XIX}^{\mathrm{e}}$ et $\mathrm{XX}^{\mathrm{e}}$ siècles, littérature comparée, linguistique contrastive, traductologie, les études sur le discours rapporté ou enfin sur la didactique du FLE. Étant donné que la plupart des contributeurs de Romanica sont des chercheurs wratislaviens, ce fait trouve certainement son reflet dans la thématique abordée dans leurs études ${ }^{20}$. En ce qui concerne les autres thèmes qui apparaissent dans la revue, leur repérage pose quelques problèmes.

${ }^{17}$ E. Kulak, op. cit.

18 J. Jakubowska-Cichoń, N. Paprocka, op. cit.

19 W. Ucherek, «L'état de l'enseignement du français à l'Université de Wrocław », Revue du Centre Européen d'Études Slaves - Enseignement du français dans les pays slaves 3, 10 février 2014, <http://etudesslaves.edel.univ-poitiers.fr/index.php?id=611> [consulté le 22.08.2017].

${ }^{20}$ Voici quelques titres de contributions publiées dans Romanica qui reflètent les thématiques en question : Krystyna Gabryjelska, «Le genre comique dans l'Encyclopédie de Diderot » (Romanica Wratislaviensia XXV, 1985) ; Małgorzata Tomicka, « Décadentisme ou symbolisme ? Réception critique des deux notions en Pologne (1886-1918)» (Romanica Wratislaviensia XX, 1983) ; Elżbieta Skibińska, «Traduire en polonais on dans sa spécificité delermienne : une tâche impossible ? (Romanica Wratislaviensia LI, 2004); Natalia Paprocka, "L'erreur en traduction dans la réflexion traductologique » (Romanica Wratislaviensia L, 2003) ; Joanna Jakubowska-Cichoń, " La mise en récit de la Grande Guerre : remarques sur l'instance narratrice dans Un long dimanche de fiançailles 
L'analyse des mots clés qui accompagnent les résumés des articles semble être un bon outil pour repérer les champs thématiques. Bien sûr, comme le constate Luc van Doorslaer, la méthode est loin d'être parfaite ${ }^{21}$. Tout d'abord, elle implique un risque d'ambiguïté terminologique et conceptuelle, étant donné que l'étiquetage relève des choix personnels des auteurs. Il serait toutefois difficile de croire qu'un système international de référence contribuerait à la clarté terminologique, puisqu'une grande majorité des internationalismes n'ont pas tout à fait les mêmes référents dans les langues vernaculaires. Qui plus est, les exemples d'études menées avec succès à partir des analyses des mots clés ${ }^{22}$ laissent supposer une efficacité assez approximative de l'outil et de son utilité pour recenser des thèmes abordés dans la revue.

La deuxième difficulté est liée au fait que dans le cas de Romanica, les mots clés n'apparaissent qu'à partir du numéro LIII (2006). Il n'est donc pas possible de retracer l'évolution des thématiques de recherche, et il ne peut être question que d'esquisser les tendances de la dernière décennie. Ainsi, sur 692 mots clés qui accompagnent les 192 articles de cette période, le mot le plus fréquent est translation. Il apparaît seul (16 occurrences), accompagné de modificateurs (du genre : audiovisual translation, translation of press articles, drama translation, litterary translation) ou enfin comme modificateur lui-même (par exemple : translation norms, translation assessment, translation's project, etc., 35 occurrences). À cela s'ajoutent les mots de la même série étymologique (translator $\times 23$, translated $\times 3$, translatology $\times 1$ ), mais aussi d'autres termes qui renvoient à différentes formes de traduction (interprétation, adaptation, compensation, etc., 122 termes au total). On pourrait donc constater que la traduction constitue un des thèmes centraux des contributeurs de la revue. Il faut cependant considérer ce résultat avec prudence, le terme pouvant se référer tout autant à l'approche traductologique qu'à celle où la traduction sert d'outil pour des objectifs spécifiques, comme par exemple la linguistique comparée ${ }^{23}$.

de Sébastien Japrisot » (Romanica Wratislaviensia LXI, 2014) ; Monika Grabowska, « Le participe présent dans la didactique du français langue étrangère » (Romanica Wratislaviensia LIV, 2007).

${ }^{21}$ Doorslaer souligne le caractère bancal des mots clés, dont le sens parfois s'imbrique et dont la division en catégories est imprécise : « [I] tis striking to see how inaccurate keywords can be, with their unnecessary overlaps, unclear division of levels etc. » (L. van Doorslaer, « Risking conceptual maps. Mapping as a keywords-related tool underlying the online Translation Studies Bibliography », Target, vol. 19, $\mathrm{n}^{\mathrm{O}} 2,2007$, p. 31).

22 Comme celle basée sur l'observation des mots clés de 12000 publications relevant de traductologie. Considérée comme un outil complémentaire, elle visait à esquisser les relations entre les concepts dans Translation Studies (voir L. van Doorslaer, « The Indicative Power of a Key Word System. A Quantitative Analysis of the Key Words in the Translation Studies Bibliography ", Meta, vol. 50, $\mathrm{n}^{\mathrm{o}} 4$ 4, 2005, <https://www.erudit.org/fr/revues/meta/2005-v50-n4-meta1024/019858ar/> [consulté le 2.08.2017]).

${ }^{23}$ Voir à ce propos E. Skibińska, « Les études romanes en Pologne : la traduction dans la recherche et dans l'enseignement ", [dans :] Les études romanes/françaises hier et aujourd'hui : 90 ans des études romanes à l'Université de Varsovie, T. Giermak-Zielińska, Z. Naliwajek et J. Żurowska 
D'autres termes qui se répètent dans Romanica témoignent d'une énorme hétérogénéité thématique. Nous y retrouvons les mots qui accompagnent les contributions à caractère linguistique (comme : préposition, address form, ou encore des termes abordant les différents aspects du discours rapporté, comme : direct speech, reported discourse, saying verbs (verbum dicendi), free indirect speech, etc., 161 termes au total), littéraire (novel, comedy, interpretation, theatre) et terminologique (terminography, terminology, lexicographic definition, 28 mots). On y observe encore parmi les mots clés des noms d'auteurs ou d'ouvrages ( 91 occurrences, dont seulement trois sont récurrentes, en l'occurrence : Voltaire, Andreï Makine, Calderón).

Nous constatons donc que les contributions publiées dans Romanica paraissent très diversifiées, et, à cause de la non-récurrence des mots clés, presque impossibles à systématiser. On observe toutefois que les termes renvoient aux disciplines qui tournent autour de divers aspects du texte, le texte lui-même étant compris dans un sens très large : il y a donc les approches qui visent son côté linguistique, son ancrage dans le contexte (littéraire, historique, discursif, etc.) ou encore celles qui se penchent sur les questions de traduction. Il est cependant frappant de constater que la majorité des termes prêtent à interprétations multiples et peuvent relever de plusieurs disciplines, comme par exemple la linguistique et la narratologie (discours rapporté), la didactique et la linguistique (address form, phonetics) ou encore la linguistique, la littérature et la traductologie (traduction). Est-ce le signe de l'imperfection du système des mots clés, qui, par sa nature généralisante, ne permet pas de bien cerner les disciplines ? Ou est-ce une preuve de la transversalité et de l'imbrication des disciplines et de l'identité " hybride » des philologues ${ }^{24}$, qui, conformément à la définition du terme dans le dictionnaire, s'adonnent à l'étude pluridimensionnelle du texte ? La réponse à cette question nécessiterait, nous semble-t-il, d'examiner le parcours de tous les romanistes qui ont contribué à la revue. Ceci étant évidemment impossible, nous avons décidé de nous limiter aux portraits de trois philologues dont les parcours diversifiés se laissent dégager de leurs publications dans Romanica ${ }^{25}$.

(dir.), Wydawnictwa Uniwersytetu Warszawskiego, Warszawa 2011, pp. 109-119 ; et J. Jakubowska-Cichoń, N. Paprocka, op. cit., p. 51.

${ }^{24}$ Voir E. Skibińska, «Between Richness and 'Non-existence': Polish Translation Researchers as a Community », [dans :] K. Taivalkoski-Shilov, L. Tiittula, M. Koponen, Communities in Translation and Interpreting, Éditions québécoises de l'œuvre, Montréal 2017, pp. 253-274.

${ }^{25}$ Le choix de trois figures masculines n'implique évidemment pas l'absence de l'apport féminin dans la recherche. Actuellement, on observe une forte féminisation du métier, ce qui se laisse voir, entre autres, dans les numéros récents de la revue. 


\subsection{TROIS BIOGRAPHIES DIFFÉRENTES}

\subsubsection{UN PHILOLOGUE AU SENS CLASSIQUE : JÓZEF HEISTEIN}

Sur les 72 publications de Józef Heistein, 21 ont paru dans Romanica Wratislaviensia. Cette « collection» impressionnante montre non seulement l'ampleur de l'activité de l'auteur, mais aussi, permet d'observer la richesse des thèmes abordés par ce chercheur. Comme le remarque Aleksander Labuda, l'activité de Heistein porte sur trois domaines différents : la littérature française, la littérature italienne et la littérature comparée ${ }^{26}$. Son intérêt pour les diverses langues et cultures romanes trouve son reflet dans les pages de la revue. On y retrouve des travaux consacrés au roman et au théâtre $\mathrm{du} \mathrm{XX}^{\mathrm{e}}$ siècle, et plus particulièrement aux tendances décadentistes, symbolistes, modernistes, futuristes, antiréalistes et avant-gardistes dans les littératures européennes. Pour le domaine italien, on observe, entre autres, des publications qui portent sur les pièces de théâtre d'Alberto Moravia ou la prose narrative de Massimo Bontempelli. Le domaine français se reflète dans des publications sur le roman absurde de Samuel Beckett ou sur le théâtre moderne d'Antonin Artaud. L'intérêt de Heistein pour la littérature comparée, enfin, trouve son reflet dans des travaux qui inscrivent les grands auteurs polonais (comme Stanisław Ignacy Witkiewicz ou Witold Gombrowicz) dans le cadre européen. Avec son goût pour les différentes littératures européennes et son aisance d'expression en langues romanes, Józef Heistein incarne parfaitement l'image « classique » du philologue ${ }^{27}$.

\subsubsection{CONSTANCE DANS L'OBJET DE LA RECHERCHE : EUGENIUSZ UCHEREK}

Auteur d'une méthode d'analyse basée sur la comparaison des textes originaux avec leurs traductions, Eugeniusz Ucherek est l'initiateur d'un courant de linguistique contrastive qui trouvera plusieurs adeptes parmi les romanistes de Wrocław à partir du milieu des années 1970. Ses recherches portent notamment sur la syntaxe des prépositions en français et en polonais ${ }^{28}$, ce qui se trouve confirmé dans les études publiées dans Romanica. Les publications d'Ucherek s'étendent sur une période de 35 ans (1972-2007). Sur 12 articles, deux seulement abordent une autre question que les prépositions, tout en s'inscrivant dans des approches structuralistes liées à la syntaxe (délimitation des groupes syntaxiques, 1972 ; syntaxe de l'article défini, 1977).

Les 10 articles restants permettent d'observer une évolution de l'objet de sa recherche. L'article de 1973, le premier où la problématique générale de la

${ }^{26}$ A. Wit Labuda, «Introduction », Romanica Wratislaviensia XLI : Mélanges de langue et de littérature offerts au Professeur Józef Heistein, Wydawnictwo Uniwersytetu Wrocławskiego, Wrocław 1996, p. 8.

${ }^{27} \mathrm{Au}$ moment de rédaction de cet article, nous avons appris avec tristesse le décès du Professeur Heistein qui s'est éteint le 12 décembre 2017.

${ }^{28}$ Voir W. Ucherek, op. cit. 
préposition est abordée, est signe avant-coureur des futurs intérêts du linguiste, qui le mèneront à élaborer sa méthode contrastive ${ }^{29}$ dont les principes seront présentés lors du Séminaire européen de l'AUPELF (le résumé de cette communication a été publié dans Romanica en 1981). Dans les années qui suivent, les contributions d'Ucherek, écrites presque toutes en français, portent sur les équivalents français des prépositions spatiales et temporelles en polonais. Les années 90 marquent un changement de perspective, l'objet des publications se tournant désormais vers les équivalents en traduction polonaise des locutions prépositionnelles françaises.

\subsubsection{OBJET DE RECHERCHE EN ÉVOLUTION : PIOTR SAWICKI}

Parfois, l'identité du philologue évolue de manière significative et implique un changement d'objet, mais aussi de culture et/ou de langue. Dès le début de sa carrière de philologue, Piotr Sawicki est à cheval entre les cultures française et espagnole. Son premier article paru dans la revue (1973) porte sur l'œuvre de Malraux consacrée à la guerre d'Espagne. Cet intérêt pour la Péninsule Ibérique se développe dans les années qui suivent. Ses recherches gravitent alors autour de la question de la guerre civile espagnole, des relations hispano-polonaises ou des récits épistolaires de voyage en Espagne (par exemple l'œuvre de Vassili Botkine). L'évolution de l'objet de recherche de Sawicki est marquée, dans Romanica, par le passage d'une langue d'expression à l'autre. Même si dans les années suivantes il reviendra encore au français, Sawicki restera toutefois fidèle à l'espagnol, la langue que semble imposer la thématique des recherches dans lesquelles il s'est spécialisé. Son amour de la culture ibérique se «matérialisera » d'ailleurs ensuite sous la forme de la revue Estudios Hispánicos dont il est le fondateur. En 2017, Sawicki a cosigné avec deux autres auteurs une monographie consacrée à l'histoire des études ibériques à l’Université de Wrocław ${ }^{30}$.

\section{EN GUISE DE CONCLUSION}

L'observation de la revue Romanica Wratislaviensia à l'occasion de son cinquantième anniversaire donne une possibilité de formuler quelques remarques sur le panorama que forment les études romanes et sur ses personnages (les contributeurs). Le profil du philologue publiant dans la revue montre le plus souvent un chercheur rattaché à une université polonaise ou, plus rarement, française.

${ }^{29} \mathrm{La}$ démarche d'Ucherek a ensuite été adoptée par d'autres chercheurs s'occupant des prépositions (voir note 14).

${ }^{30}$ P. Sawicki, M. Minkiewicz, A. Olchówka, Iberystyka wrocławska (1967-2007). Ilustrowany przewodnik retrospektywny, Atut, Wrocław 2017. 
Romanica n'est cependant pas refermée sur le milieu national, et le nombre de volumes réunissant des actes de colloques internationaux en est la preuve.

L'image du philologue qui se dégage de Romanica semble rompre avec la définition classique du terme. Avec l'autonomisation des recherches et l'intérêt porté à des aspects choisis des textes (le plus souvent contemporains) ainsi que, dans la majorité des cas, une seule langue d'expression, le philologue d'aujourd'hui répond plutôt à l'image du néo-philologue qu'à celle du philologue au sens classique du terme. Les thématiques qu'il entreprend sont très diverses. Comme le montrent les mots clés, elles s'inscrivent dans les différentes branches des études philologiques. Cela semble plutôt un signe de la transversalité des disciplines que de l'identité hybride des chercheurs. Certes, l'analyse de quelques carrières personnelles montre qu'il y a des chercheurs qui s'inscrivent dans l'image classique du philologue. Mais il y en a aussi qui restent fidèles à une problématique assez circonscrite. Ou encore, qui sont à la recherche de leur centre d'intérêt et de sa langue d'expression. Bref, il n'existe pas de modèle unique d'évolution du philologue. Si l'observation des recherches publiées dans Romanica Wratislaviensia permet de constater une certaine évolution de l'identité professionnelle, et même une sorte de rupture avec l'image classique du philologue, les évolutions et les trajectoires personnelles restent en revanche aussi diversifiées que les contributeurs.

\section{TOWARDS A CARTOGRAPHY OF PHILOLOGICAL RESEARCH: OBSERVATIONS ON THE FIFTIETH ANNIVERSARY OF THE REVUE ROMANICA WRATISLAVIENSIA}

\section{Summary}

The paper presents an overview of romanistic research on the occasion of Romanica Wratislaviensia's fiftieth anniversary. In as much as the revue has undergone several transformations over the years, the Wratislavian romanists consequently could not escape a certain evolution. The transition of their domains of research, their careers, all these changes are inscribed on the pages of Romanica Wratislaviensia. Therefore, the aim of the article is to sketch the landscape of philological research which emerges from the contributions published in the revue, from its founding in 1968 to its last issue in 2016. We will consider the extent to which the identity of a philologist emerging from the numerous publications in Romanica Wratislaviensia fits with the historical definition of a philologist.

Key words: philologist, identity, Romanica Wratislaviensia. 